\title{
Determinación del contenido de cemento en morteros y hormigones fraguados: datos para la puesta al día del método
}

JOSE CALleja* y CESAR DEL OLMO**

\section{resumern}

La determinación de la dosis de cemento de un conglomerado se basa, en general, en unas hipótesis respecto de los materiales, y en un valor medio estimado, en cuanto al contenido de sílice soluble del cemento empleado.

Este contenido medio está sujeto a variación, lógicamente unidireccional, a medida que en la práctica se exige más de los conglomerantes y se dispone de medios de fabricación más perfectos para mejorar su calidad.

A base de una amplia estadística al día, sobre 600 resultados analíticos de cementos designados como portland que cubren el período de 1963 a 1969, se demuestra que el valor medio fijado por la norma NELC 5.01-a (2), ya anticuada, debe ser modificado en el sentido de sustituir el 20,5\% aún hoy vigente, por el 19,5\%, más en consonancia con la realidad.

El mantenimiento del 20,5 \% puede perjudicar en una cierta medida mayor o menor, según los casos, a los constructores de buena fe que emplean en sus dosificaciones el cemento estipulado.

\section{INTRODUCCION}

La solución al problema de averiguar el contenido de cemento con que en su día fue hecho un mortero $u$ hormigón, ya fraguado y endurecido, tiene una gran importancia técnica. La razón es que dicha solución se toma como base para enjuiciar el material y decidir si cumple o no con las condiciones de proyecto, en cuanto se refiere a dosificación. El planteamiento se hace casi siempre con objeto de fijar responsabilidades en caso de incumplimiento, lo cual tiene siempre repercusiones económicas, y muchas veces tambien de otro tipo, cuando el asunto es suscitado por fallos de obras, estructuras o elementos de construcción, con daños de mayor cuantía, materiales y personales.

Como casi siempre, la solución al problema es fácil y muy aproximada a la real y verdadera, cuando se dispone de los datos necesarios y suficientes para el planteamiento, o

* Doctor en Ciencias Químicas. Investigador Científico del C.S.I.C. Jefe del Departamento de Química del I.E.T.c.c. (Patronato "Juan de la Cierva" de Investigación Científica y Técnica).

** Licenciado en Ciencias Químicas. Colaborador en el Departamento de Química del I.E.T.c.c. 
cuando se cuenta con los elementos necesarios para extraer de ellos estos datos. Por desgracia, esta situación ideal y más favorable no se da casi nunca en la práctica.

Por ello se trata de suplir, con mejor voluntad que éxito, los datos reales que faltan, por otros que se adoptan con mayor o menor justificación en cada caso, implicando tales sustituciones la introducción de mayores errores en los resultados.

Los errores son, en general, varios, y reconocen diversos orígenes, desde la falta de representatividad de la muestra de material que se enjuicia, hasta los que responden a la citada suplencia de datos, pasando por los más normales, propios de los métodos analíticos que se emplean. En todo caso, los errores, que según su sentido pueden actuar a favor o en contra de la decisión o el juicio justos, deben reducirse al mínimo. Respecto de estos y otros errores, así como de su importancia y repercusión, ya se ha tratado en otra ocasión (1).

\section{INSTRUCCIONES Y NORMAS}

Desde hace años se viene utilizando para determinar la dosificación de hormigones y morteros fraguados, fabricados con cemento, la norma NELC 5.01-a (2). Según ella, la Instrucción para el Proyecto y Ejecución de Obras de Hormigón (3), vigente al ser publicada dicha norma, "no señalaba método alguno para investigar la dosificación empleada en la preparación de morteros y hormigones de cemento". Tampoco indica nada al respecto la Instrucción para el Proyecto y Ejecución de Obras de Hormigón en Masa o Armado, de reciente publicación y vigencia (4). Así, pues, la norma NELC 5.01-a, que vino a suplir una carencia de la Instrucción antigua (3), no ha sido recogida por la moderna (4), pese a lo cual, y por el momento, se sigue aplicando a la resolución de problemas de dosificación.

Aparte de esto, en la actualidad existe la correspondiente norma ASTM (5) como más difundida, además de otras, locales o domésticas, vigentes en los respectivos países de origen. Con carácter más general, y en fase final de elaboración por parte de un organismo internacional, el CEMBUREAU, hay un método en cuya puesta a punto, y como miembro de dicho organismo, ha tomado parte España, a través del I.E.T.c.c. (6).

Finalmente, existe un método, simultáneo al precedentemente citado en cuanto a su desarrollo, que sobre éste y todos los demás posee, entre otras ventajas, la de estar libre de hipótesis y tener, por lo tanto, una validez general absoluta. Utiliza, además, no uno -ni dos, como la norma ASTM (5) - sino todos los parámetros utilizables, con lo que su fiabilidad es máxima. Es un método de concepción y factura netamente españolas (7).

\section{CARACTERISTICAS Y CAMPOS DE VALIDEZ DE LOS METODOS EN USO}

La norma española NELC 5.01-a puntualiza que "es aplicable a cualquier mortero y hormigón que no contenga materiales silíceos atacables por los ácidos, tales como escorias granuladas de altos hornos, puzolanas, etc.", con lo cual "sólo será aplicable a los morteros $\mathrm{u}$ hormigones fabricados con cemento portland, y a condición de adoptar un valor medio para la sílice de los cementos portland españoles", salvo que se conozca "la riqueza en sílice del cemento empleado", en cuyo caso "la norma podrá aplicarse, cualquiera que sea la naturaleza o calidad del cemento".

Dado que, como se indicaba en la Introducción, en general no se conoce la riqueza en sílice del cemento empleado, ni se dispone de muestras del mismo para determinarla, el 
campo de aplicación del método queda limitado a los morteros y hormigones hechos con cemento portland.

La antigua norma española para cementos (8) definía el cemento portland como "producto reducido a polvo fino que se obtiene con la calcinación, hasta un principio de fusión, de mezclas muy íntimas, artificialmente hechas y convenientemente dosificadas, de materias calizas y arcillosas, sin más adición que la de yeso, que no podrá exceder de $3 \%$ ". Con esto, la norma NELC 5.01-a era aplicable en todos los casos para la determinación de la dosificación de cemento en los hormigones y morteros hechos con cemento portland.

La norma española actualmente vigente para cementos (9) define a los portland como "conglomerantes hidráulicos que se obtienen por pulverización del clínker, y sin más adición que la de piedra de yeso natural"; pero añade la siguiente ampliación de la definición: "Eventualmente, puede darse la denominación comercial de cementos portland a aquéllos que, además de los componentes principales, clínker y piedra de yeso, contengan otras adiciones no nocivas en proporción inferior al $10 \%$, con objeto de mejorar algunas de las cualidades de los conglomerantes o de los morteros y hormigones con ellos fabricados, siempre que los cementos resultantes cumplan todas las condiciones químicas, físicas y mecánicas que se especifican para el portland". Con esto, no siempre será aplicable la norma NELC 5.01-a para resolver correctamente problemas de dosificación de cemento en hormigones y morteros, aunque estén fabricados con cemento portland.

En efecto, la adición del $10 \%$ puede ser tal que contenga sílice soluble, o que no la contenga. Para fijar ideas, se van a considerar los dos casos extremos posibles, entendiéndose que cualquiera de los casos prácticos reales se hallará comprendido entre ambos: el $10 \%$ de adición es en su totalidad sílice soluble; el $10 \%$ de adición no contiene en absoluto sílice soluble.

En el primer caso, el "conglomerante" constará del $90 \%$ de cemento portland y de $10 \%$ de sílice soluble. Si se admite para el primero un contenido de $20,5 \%$ de sílice soluble, el total de ésta en el "conglomerante" será: $10 \%+(90 \% \times 20,5 \%)=28,45 \%$. En el segundo caso dicho total será: $90 \% \times 20,5 \%=18,45 \%$. Es decir, las cantidades de sílice soluble (y de cemento por metro cúbico de hormigón) halladas en ambos casos estarán en la relación 28,45 : 18,45 = 1,54. En efecto, un hormigón que tenga el $13 \%$ en peso de "conglomerante" tendrá, en el primer caso: $13 \times 28,45: 100=3,70 \%$ de sílice soluble; y en el segundo caso: $13 \times 18,45: 100=2,40 \%$ de sílice soluble. Si el $13 \%$ no fuera de "conglomerante", sino de cemento portland sin aditivo alguno, el hormigón tendría: $13 \times$ $\times 20,5: 100=2,67 \%$ de sílice soluble (tercer caso).

Según la norma NELC 5.01-a, las dosificaciones halladas para un hormigón de densidad 2,30 serían:

En el primer caso:

$$
\frac{3,70 \times 2,30 \times 1.000}{28,45}=300 \mathrm{~kg} / \mathrm{m}^{3}
$$

En el segundo caso:

$$
\frac{2,40 \times 2,30 \times 1.000}{18,45}=300 \mathrm{~kg} / \mathrm{m}^{3}
$$


En el tercer caso:

$$
\frac{2,67 \times 2,30 \times 1.000}{20,5}=300 \mathrm{~kg} / \mathrm{m}^{3}
$$

Coincidiendo en todos ellos con la dosificación verdadera del hormigón:

$$
(13: 100)(2,3 \times 1.000)=300 \mathrm{~kg} / \mathrm{m}^{3}
$$

Pero, de no conocerse los contenidos de sílice soluble del conglomerante y tener que emplear el valor medio del 20,5 \% de la norma NELC 5.01-a, las dosificaciones serían otras, a saber:

En el primer caso:

$$
\frac{3,70 \times 2,30 \times 1.000}{20,5}=415 \mathrm{~kg} / \mathrm{m}^{3}
$$

En el segundo caso:

$$
\frac{2,40 \times 2,30 \times 1.000}{20,5}=269 \mathrm{~kg} / \mathrm{m}^{3}
$$

En el tercer caso:

$$
\frac{2,67 \times 2,30 \times 1.000}{20,5}=300 \mathrm{~kg} / \mathrm{m}^{3}
$$

coincidente con la correspondiente anterior y con la verdadera.

De esto se deduce que las adiciones silícicas (primer caso) pueden inducir a un error máximo del orden de $(415-300) \cdot 100: 300=38 \%$ por exceso. También se deduce que las adiciones no silícicas (caso segundo) no impiden obtener la dosificación verdadera, en cuanto a contenido real de cemento portland en el hormigón $(300 \times 90: 100=270 \mathrm{~kg} /$ $\left./ \mathrm{m}^{3}\right)$; en cuanto a contenido de "conglomerante", dan lugar a un error máximo del orden de $(300-270) 100: 300=10 \%$ por defecto, si el "conglomerante" se ajusta a la definición ampliada de cemento portland, según la norma española (9).

Estos errores son independientes de los propios del método, los cuales pueden llegar a ser, según la norma NELC 5.01-a, del orden de $\pm 4 \%$ si se conoce o determina la sílice soluble del cemento, y del orden de $\pm 18 \%$ si no se conoce. Es interesante justificar numéricamente estos errores. En cuanto al de $\pm 4 \%$, la norma ASTM para el análisis químico del cemento portland (10) admite una diferencia máxima de 0,24 entre valores extremos de tres determinaciones de sílice soluble. Con esto, el contenido medio verdadero de sílice del cemento, de $20,5 \%$ en el ejemplo anterior, puede oscilar entre 20,26 y 20,74 $\%$; de igual manera, el contenido medio de sílice del hormigón, de $2,67 \%$, puede oscilar entre 2,646 y $2,694 \%$. Si se admite al mismo tiempo como lógico que la densidad del hormigón de 2,30 puede estar afectada de un error que la haga oscilar entre 2,25 y 2,35, se 
tendrán diversas posibilidades, de las cuales, las extremas y más desfavorables darían dosificaciones de

$$
\frac{2,646 \times 2,25 \times 1.000}{20,74}=287 \mathrm{~kg} / \mathrm{m}^{3}
$$

y de

$$
\frac{2,694 \times 2,35 \times 1.000}{20,26}=312 \mathrm{~kg} / \mathrm{m}^{3}
$$

lo cual supone una diferencia máxima de 25 en 300 , es decir, del orden de $8 \%$, esto es, de $\pm 4 \%$.

En cuanto al error del $18 \%$, éste incluye el anterior (determinación de densidad y de sílice soluble en el hormigón), y además el derivado de tomar un valor medio, en lugar del valor real (con su error), para el contenido de sílice soluble del cemento, como consecuencia de no conocerse dicho valor, ni poderse determinar por carecer de muestra de cemento. En efecto, supuesto un campo de variación del contenido de sílice del cemento, comprendido entre 17,5 y $23,5 \%$ (intervalo razonable), para dosificaciones de $150,200,250$, $300,350,400$ y $450 \mathrm{~kg}$ de cemento por metro cúbico de hormigón, y partiendo de la fórmula de dosificación [I] que da la norma NELC 5.01-a:

$$
\mathrm{D}=1.000 \frac{\mathrm{S}_{\mathrm{h}} \cdot \mathrm{d}_{\mathrm{h}}}{\mathrm{S}_{\mathrm{c}}}
$$

en la que:

$$
\begin{aligned}
& \mathrm{D}=\text { dosificación de cemento en el hormigón, en } \mathrm{kg} / \mathrm{m}^{3} ; \\
& \mathrm{d}_{\mathrm{h}}=\text { densidad del hormigón, en } \mathrm{kg} / \mathrm{dm}^{3} ; \\
& \mathrm{S}_{\mathrm{h}}=\text { tanto por ciento de sílice soluble del hormigón; } \\
& \mathrm{S}_{\mathrm{c}}=\text { tanto por ciento de sílice soluble del cemento, }
\end{aligned}
$$

se tendrá entonces, para un hormigón de densidad $d_{h}=2,30 \mathrm{~kg} / \mathrm{dm}^{3}$, que:

$$
\mathrm{D} \cdot \mathrm{S}_{\mathrm{c}}=2.300 \cdot \mathrm{S}_{\mathrm{h}}
$$

La tabla $1 \mathrm{da}$, en I los distintos valores del producto $\mathrm{D} \cdot \mathrm{S}_{\mathrm{c}}$ en los casos supuestos, y en II, los valores de $\mathrm{S}_{\mathrm{h}}$ deducidos de los anteriores, según [II], en cada caso.

Si no se conoce el valor de $\mathrm{S}_{\mathrm{c}}$, al calcular la dosificación con la fórmula [I] hay que utilizar para $S_{c}$ el valor 20,5, con lo cual se obtienen dosificaciones $D_{M}$ dadas por la fórmula [III] :

$$
\mathrm{D}_{\mathrm{M}}=\frac{2.300}{20,5} \cdot \mathrm{S}_{\mathrm{h}}=112,2 \cdot \mathrm{S}_{\mathrm{h}}
$$

Estas dosificaciones, calculadas con los valores $\mathrm{S}_{\mathrm{h}}$ de la tabla 1-II, son las dadas en la tabla 1-III.

La tabla 1-IV da las diferencias, con su signo, entre las dosificaciones calculadas $D_{M}$ de la tabla 1-III y las dosificaciones $\mathrm{D}$ nominales: $\mathrm{D}_{\mathrm{M}}-\mathrm{D}$. 
T A B L A 1

$\mathrm{D} \cdot \mathrm{S}_{\mathrm{c}}=2.300 \cdot \mathrm{S}_{\mathrm{b}}$

Dosificación nominal D del hormigón

$\mathbf{k g} / \mathbf{m}^{3}$

\begin{tabular}{|c|c|c|c|c|c|c|c|}
\hline 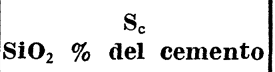 & 150 & 200 & 250 & 300 & 350 & 400 & 450 \\
\hline 17,5 & 2.625 & 3.500 & 4.375 & 5.250 & 6.125 & 7.000 & 7.875 \\
\hline 20,5 & 3.075 & 4.100 & 5.125 & 6.150 & 7.175 & 8.200 & 9.225 \\
\hline 23,5 & 3.525 & 4.700 & 5.875 & 7.050 & 8.225 & 9.400 & 10.575 \\
\hline
\end{tabular}

\begin{tabular}{|c|c|c|c|c|c|c|c|}
\hline & \multicolumn{7}{|c|}{ Sílice soluble $\mathrm{S}_{\mathrm{h}} \%$ del hormigón } \\
\hline 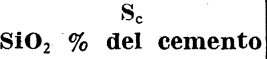 & 150 & 200 & 250 & 300 & 350 & 400 & 45 \\
\hline 17,5 & 1,14 & 1,52 & 1,90 & 2,28 & 2,66 & 3,04 & $3,4:$ \\
\hline 20,5 & 1,34 & 1,78 & 2,23 & 2,67 & 3,12 & 3,57 & 4,0 \\
\hline & & & & 2 & & & \\
\hline
\end{tabular}

\begin{tabular}{|c|c|c|c|c|c|c|c|}
\hline \multirow[b]{2}{*}{$\begin{array}{llrl} & & \mathbf{S}_{\mathrm{c}} & \\
\mathbf{S i O}_{2} & \% & \text { del } & \text { cemento }\end{array}$} & \multicolumn{5}{|c|}{ Dosificaciones $D_{M}$ calculadas } & \multicolumn{2}{|c|}{$\mathbf{k g} / \mathbf{m}^{3}$} \\
\hline & 150 & 200 & 250 & 300 & 350 & 400 & 450 \\
\hline 17,5 & 128 & 171 & 213 & 256 & 298 & 341 & 384 \\
\hline 20,5 & 150 & 200 & 250 & 300 & 350 & 401 & 450 \\
\hline 23,5 & 172 & 229 & 286 & 344 & 402 & 459 & 516 \\
\hline
\end{tabular}

\begin{tabular}{|c|c|c|c|c|c|c|c|}
\hline \multirow[b]{2}{*}{$\mathrm{SiO}_{2} \% \quad \begin{array}{r}\mathrm{S}_{\mathrm{c}} \\
\mathrm{del}\end{array}$} & \multicolumn{7}{|c|}{ Diferencias $\mathbf{D}_{M}-\mathbf{D}$} \\
\hline & 150 & 200 & 250 & 300 & 350 & 400 & 450 \\
\hline 17,5 & -22 & -29 & -37 & -44 & -52 & -59 & -66 \\
\hline 20,5 & 0 & 0 & 0 & 0 & 0 & 0 & 0 \\
\hline 23,5 & +22 & +29 & +36 & +44 & +52 & +59 & +66 \\
\hline
\end{tabular}

\begin{tabular}{|c|c|c|c|c|c|c|c|}
\hline \multirow[b]{2}{*}{\begin{tabular}{lll} 
& \multicolumn{3}{c}{$\mathbf{S}_{\mathrm{c}}$} \\
$\mathrm{SiO}_{2}$ & $\%$ & del cemento
\end{tabular}} & \multicolumn{7}{|c|}{ Errores por ciento E \% } \\
\hline & 150 & 200 & 250 & 300 & 350 & 400 & 450 \\
\hline 17,5 & -15 & $-14,5$ & -15 & -15 & -15 & -15 & -15 \\
\hline 20,5 & 0 & 0 & 0 & 0 & 0 & 0 & 0 \\
\hline 23,5 & +15 & $+14,5$ & $+14,5$ & +15 & +15 & +15 & +15 \\
\hline
\end{tabular}


La tabla 1-V da los errores relativos $\mathrm{E}$ en tanto por ciento, con su signo, calculados según la fórmula:

$$
\mathrm{E} \%=100 \frac{\mathrm{D}_{\mathrm{M}}-\mathrm{D}}{\mathrm{D}}
$$

Como puede apreciarse, estos errores oscilan alrededor del $\pm 15 \%$, que sumado al $\pm 4 \%$ hallado anteriormente da $\pm 19 \%$, esto es, $\pm 18 \%$, teniendo en cuenta las aproximaciones hechas en los cálculos.

Un error de $\pm 18 \%$ atribuible sólo al valor medio tomado para la sílice del cemento implicaria, para un hormigón de $300 \mathrm{~kg} / \mathrm{m}^{3}$ de dosificación nominal $\mathrm{D}$, una diferencia de $\pm 54 \mathrm{~kg}$, es decir, unas dosificaciones calculadas extremas $D_{\mathrm{M}}$ de $246 \mathrm{~kg}$ y $354 \mathrm{~kg}$, respectivamente; valores que según la fórmula [III] dan para $\mathrm{S}_{\mathrm{h}} 2,19 \%$ y $3,16 \%$, los que a su vez, y según la fórmula [II], dan para $\mathrm{S}_{c} 16,8$ y $24,2 \%$ respectivamente, es decir $17 \%$ y $24 \%$, valores muy próximos a los $17,5 \%$ y $23,5 \%$ considerados antes. Esto quiere decir que el error que se comete al tomar un valor medio para la sílice soluble del cemento, en lugar de tomar su valor real, es mucho mayor (del orden de cuatro veces) que el propio del método.

Es evidente que los errores descritos, y sobre todo los debidos a las posibles adiciones silícicas al clínker, pueden inducir a calificar como bien dosificado un hormigón que en realidad no lo esté, con la consiguiente equivocación en el enjuiciamiento del material y todas sus consecuencias. La conclusión de todo ello es que, la norma NELC 5.01-a no es aplicable con carácter general a la dosificación de cemento portland en hormigones y morteros fraguados hechos con este material, a menos que se conozca el contenido de sílice soluble del cemento empleado, o se disponga de muestra del mismo para determinarla.

Es decir, dada la definición ampliada que de cemento portland da la vigente norma española para conglomerantes, éste queda sujeto, en general, a las mismas limitaciones que establece la norma NELC 5.01-a para los siderúrgicos y puzolánicos, en cuanto a determinación de la dosificación de morteros y hormigones fraguados, esto es, a conocer la sílice soluble del conglomerante, o a poderla determinar disponiendo de muestra para ello.

\section{ACTUALIZACION DE LA NORMA NELC 5.01-a}

Otro aspecto a considerar en la norma NELC 5.01-a es el de la justificación del 20,5 \%, tomado como valor medio del contenido de sílice soluble de los cementos españoles. Es posible que en la fecha en que se redactó esta norma -1947- fuera ese, en efecto, el valor medio. Pero la composición de los cementos portland, al igual que su producción, ha cambiado bastante con el tiempo, particularmente en España. Es posible también que, a falta de datos, se tomase dicho valor por analogía con la correspondiente norma norteamericana que entonces (11), como ahora (5) admite para los cementos portland un contenido medio de sílice soluble de $21 \%$.

Se ha manifestado en España, a lo largo del tiempo, una tendencia a elevar el módulo hidráulico y el grado de saturación de los cementos, aumentando el contenido de cal y disminuyendo en proporción los contenidos de los constituyentes ácidos o factores hidráulicos. Pero, además, la tendencia simultánea a reducir (en algunos casos) el contenido de alúmina, se ha visto compensada por el incremento paralelo del contenido de óxido férrico, con lo cual, al quedar prácticamente constante la suma de los sesquióxidos, lo que 
ha sucedido, en definitiva, es que, en general, el contenido de sílice de los cementos portland españoles ha disminuido. Esta apreciación cualitativa es aceptable, sin más. Con ello surge la necesidad de revisar ese valor medio del $20,5 \%$, que en la actualidad deberá ser más bajo, al contrario que en las normas norteamericanas (11) y (5), si bien esta revisión, y la adopción del nuevo valor medio actualizado, deberá ser el resultado de un estudio cuantitativo efectuado correctamente sobre datos reales.

Con esta finalidad se ha llevado a cabo una estadística, tomando como base los cementos que, bajo la denominación de portland, o sin denominación distinta de ésta, se han ensayado y analizado en el Departamento de Química del I.E.T.c.c. durante los años 1963 a 1969, ambos inclusive. El número de cementos asciende a 601 , con contenidos de sílice que oscila entre un mínimo de $12,8 \%$ y un máximo de $25,9 \%$.

En la tabla 2 se dan todos los valores agrupados por intervalos de clase I, así como los valores centrales $X_{i}$ de dichos intervalos, su frecuencia $N_{i}$ y los productos de ambas $X_{i} N_{i}$ (columnas 1, 2, 3 y 4). La distribución responde a la ley de Gauss-Laplace, como pone de relieve la figura 1. La figura 2 da la variación de los valores medios de la sílice soluble, por años, en el período señalado, para las distintas categorías de cementos portland.

Las columnas 5,6 y 7 de la tabla 2 contienen respectivamente las diferencias entre la media $\overline{\mathrm{X}}$ y cada valor central $\mathrm{X}_{\mathrm{i}}, \Delta_{\mathrm{i}}=\left(\overline{\mathrm{X}}-\mathrm{X}_{\mathrm{i}}\right)$, los cuadrados de estas diferencias $\Delta_{\mathrm{i}}{ }^{2}$, y los productos de estos cuadrados por las correspondientes frecuencias $\mathrm{N}_{\mathrm{i}} \Delta_{\mathrm{i}}{ }^{2}$.

A partir de estos valores se calculan las sumas $\Sigma \mathrm{N}_{\mathrm{i}}, \Sigma \mathrm{X}_{\mathrm{i}} \cdot \mathrm{N}_{\mathrm{i}}$ y $\Sigma \mathrm{N}_{\mathrm{i}} \Delta_{\mathrm{i}}{ }^{2}$, con los que se calculan a su vez la desviación standard $\sigma$, el coeficiente de variación $\mathrm{V} \%$, y el intervalo de confianza $\overline{\mathrm{X}} \pm \sigma \cdot \mathrm{t}$ para niveles de probabilidad de $95 \%(\mathrm{t}=1,96)$ así como errores probables absolutos $\varepsilon$ y relativos $\varepsilon \%$ sobre la media. Se tienen así los parámetros calculados dados en la primera línea de la tabla 3 .

Como es fácil apreciar, el intervalo de confianza $\overline{\mathrm{X}} \pm \sigma \cdot \mathrm{t}$, para una probabilidad de 95 $\%$, señala como valores aberrantes los superiores a 22,14 y los inferiores a 16,86 . Descartados éstos y calculada la nueva media, así como los restantes parámetros, se tienen los valores dados en la línea segunda de la tabla 3, pudiéndose apreciar que sigue habiendo valores aberrantes.

Si se eliminan éstos por el procedimiento descrito, tantas veces como sea necesario, se llega a los sucesivos resultados de las líneas $3,4,5$ y 6 de la tabla 3 , los cuales están representados por los acotamientos señalados en la tabla $2 \mathrm{y}$ en la figura 1.

Se aprecia que la serie inicial de resultados abarca un campo de variación desde $12,8 \%$ hasta $25,9 \%$, mientras que en las series sucesivamente mantenidas, una vez descartados los resultados aberrantes, la variación llega a ser sólo de $18,1 \%$ a 20,8 \% (en la última serie). Este último margen es mucho menos amplio que el que se ha calculado en 3 para un error máximo de $\pm 18 \%$.

Por otra parte, se puede observar en la tabla 3 que, en las sucesivas eliminaciones de valores aberrantes, apenas varían ni la media ni los errores. Estos pueden llegar a ser tales que, como máximo, y según la fórmula de dosificación [I], den valores comprendidos entre

$$
\mathrm{D}_{\mathrm{M}}=1.000 \frac{2,67 \times 2,30}{19,5}=315
$$


T A

\begin{tabular}{|c|c|c|c|c|c|c|c|c|c|c|c|c|c|}
\hline $\begin{array}{c}\text { Intervalos de } \\
\text { clases } \\
\text { I }\end{array}$ & 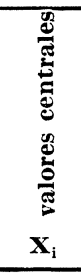 & 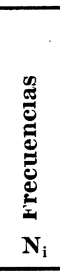 & 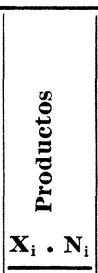 & 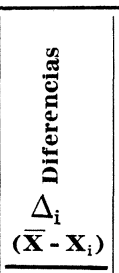 & 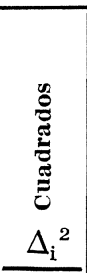 & 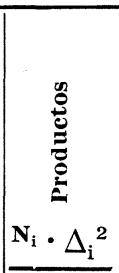 & $\begin{array}{c}\text { Intervalos de } \\
\text { clases } \\
\text { I }\end{array}$ & 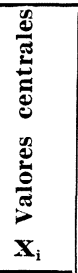 & 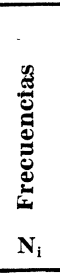 & 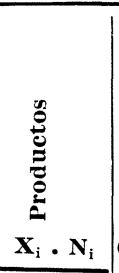 & 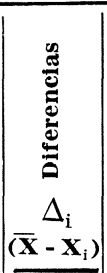 & 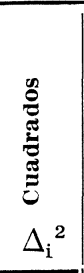 & 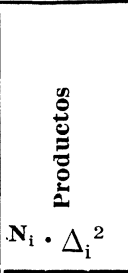 \\
\hline $25,85-25,95$ & 25,9 & 1 & 25,9 & 6,4 & 40,96 & 40,96 & $19,45-19,55$ & $\overline{19,5}$ & 26 & 507,0 & 0,0 & 0,00 & 0,00 \\
\hline \multirow[t]{35}{*}{$25,05-25,15$} & 25,1 & 1 & 25,1 & 5,6 & 31,36 & 31,36 & & $\overline{19,4}$ & 28 & 543,2 & 0,1 & 0,01 & 0,28 \\
\hline & 25,0 & 1 & 25,0 & 5,5 & 30,25 & 30,25 & & 19,3 & 18 & 347,4 & 0,2 & 0,04 & 0,72 \\
\hline & 23,8 & 1 & 23,8 & 4,3 & 18,49 & 18,49 & & $\overline{19,2}$ & 23 & 441,6 & 0,3 & 0,09 & 2,07 \\
\hline & 23,7 & 1 & 23,7 & 4,2 & 17,64 & 17,64 & & 19,1 & 18 & 343,8 & 0,4 & 0,16 & 2,88 \\
\hline & 23,2 & 3 & 69,6 & 3,7 & 13,69 & 41,07 & & 19,0 & 24 & 456,0 & 0,5 & 0,25 & 6,00 \\
\hline & 23,1 & 1 & 23,1 & 3,6 & 12,96 & 12,96 & & $\overline{18,9}$ & 22 & 415,8 & 0,6 & 0,36 & 7,92 \\
\hline & 22,9 & 1 & 22,9 & 3,4 & 11,56 & 11,56 & & 18,8 & 17 & 319,6 & 0,7 & 0,49 & 8,33 \\
\hline & 22,3 & 2 & 44,6 & 2,8 & 7,84 & 15,68 & & 18,7 & 15 & 280,5 & 0,8 & 0,64 & 9,60 \\
\hline & 22,2 & 1 & 22,2 & 2,7 & 7,29 & 7,29 & & $\overline{18,6}$ & 19 & 353,4 & 0,9 & 0,81 & 15,39 \\
\hline & 22,1 & 2 & 44,2 & 2,6 & 6,76 & 13,52 & & 18,5 & 13 & 240,5 & 1,0 & 1,00 & 13,00 \\
\hline & 22,0 & 4 & 88,0 & 2,5 & 6,25 & 25,00 & & $\overline{18,4}$ & 7 & 128,8 & 1,1 & $\overline{1,21}$ & 8,47 \\
\hline & 21,9 & 3 & 65,7 & 2,4 & 5,76 & 17,28 & & 18,3 & 6 & 109,8 & 1,2 & 1,44 & 8,64 \\
\hline & 21,8 & 1 & 21,8 & 2,3 & $\overline{5,29}$ & 5,29 & & $\overline{18,2}$ & 6 & 109,2 & 1,3 & 1,69 & 10,14 \\
\hline & 21,7 & 7 & 151,9 & 2,2 & 4,84 & 33,88 & & 18,1 & 9 & 162,9 & 1,4 & 1,96 & 17,64 \\
\hline & 21,6 & 3 & 64,8 & 2,1 & 4,41 & 13,23 & & 18,0 & 5 & 90,0 & 1,5 & $\overline{2,25}$ & 11,25 \\
\hline & 21,5 & 6 & 129,0 & 2,0 & 4,00 & 24,00 & & 17,9 & 4 & 71,6 & 1,6 & $\overline{2,56}$ & 10,24 \\
\hline & 21,4 & 6 & 128,4 & 1,9 & 3,61 & 21,66 & & 17,8 & 4 & 71,2 & 1,7 & 2,89 & 11,56 \\
\hline & 21,3 & 8 & 170,4 & 1,8 & 3,24 & 25,92 & & 17,7 & 9 & 159,3 & 1,8 & 3,24 & 29,16 \\
\hline & 21,2 & 3 & 63,6 & 1,7 & 2,89 & 8,67 & & 17,6 & 6 & 105,6 & 1,9 & 3,61 & 21,66 \\
\hline & 21,1 & 5 & 105,5 & 1,6 & 2,56 & 12,80 & & 17,5 & 6 & 105,0 & 2,0 & 4,00 & 24,00 \\
\hline & 21,0 & 8 & 168,0 & 1,5 & 2,25 & 18,00 & & 17,4 & 7 & 121,8 & 2,1 & 4,41 & 30,87 \\
\hline & 20,9 & 6 & 125,4 & 1,4 & 1,96 & 11,76 & & 17,3 & 4 & 69,2 & 2,2 & 4,84 & 19,36 \\
\hline & 20,8 & 8 & 166,4 & 1,3 & 1,69 & 13,52 & & 17,2 & 4 & 68,8 & 2,3 & 5,29 & 21,16 \\
\hline & 20,7 & 5 & 103,5 & 1,2 & 1,44 & 7,20 & & 17,1 & 1 & 17,1 & 2,4 & 5,76 & 5,76 \\
\hline & 20,6 & 15 & 309,0 & 1,1 & 1,21 & 18,15 & & 17,0 & 1 & 17,0 & 2,5 & 6,25 & 6,25 \\
\hline & 20,5 & 7 & 143,5 & 1,0 & 1,00 & 7,00 & & 16,9 & 4 & 27,6 & 2,6 & 6,76 & 27,04 \\
\hline & 20,4 & 14 & 285,6 & 0,9 & 0,81 & 11,34 & & 16,6 & 2 & 33,2 & 2,9 & $\overline{8,41}$ & 16,82 \\
\hline & 20,3 & 12 & 243,6 & 0,8 & 0,64 & 7,68 & & 16,2 & 1 & 16,2 & 3,3 & 10,89 & 10,89 \\
\hline & 20,2 & 17 & 343,4 & 0,7 & 0,49 & 8,33 & & 16,0 & 1 & 16,0 & 3,5 & 12,25 & 12,25 \\
\hline & 20,1 & 16 & 321,6 & 0,6 & 0,36 & 5,76 & & 14,3 & 1 & 14,3 & 5,2 & 27,04 & 27,04 \\
\hline & 20,0 & 17 & 340,0 & 0,5 & 0,25 & 4,25 & & $\overline{14,2}$ & 1 & 14,2 & 5,3 & 28,09 & 28,09 \\
\hline & 19,9 & 25 & 497,5 & 0,4 & 0,16 & 4,00 & & 13,7 & 1 & 13,7 & 5,8 & 33,64 & 33,64 \\
\hline & 19,8 & 32 & $\begin{array}{l}633,6 \\
\end{array}$ & 0,3 & 0,09 & 2,88 & & 13,6 & 1 & 13,6 & 5,9 & $\overline{34,81}$ & 34,81 \\
\hline & 19,7 & 24 & 472,8 & 0,2 & 0,04 & 0,96 & & 12,8 & 1 & 12,8 & 6,7 & 44,89 & 44,89 \\
\hline & 19,6 & 19 & 372,4 & 0,1 & 0,01 & 0,19 & $\Sigma$ & & 601 & $11.723,2$ & & & $1.087,35$ \\
\hline
\end{tabular}

Observación: Para no alargar demasiado la tabla se han suprimido los intervalos de clase con frecuencia cero. 
T A

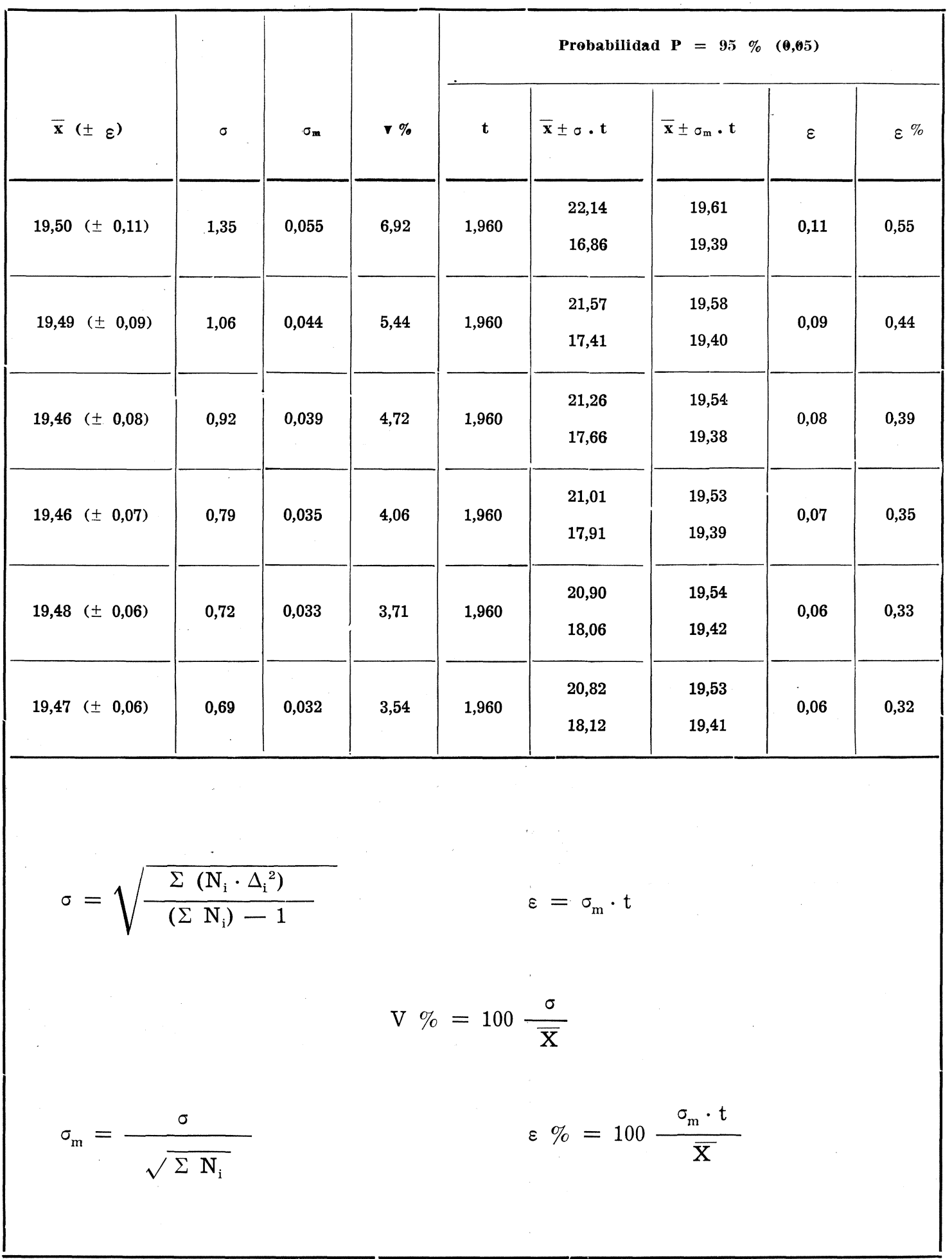


y

$$
\mathrm{D}=\left\{\begin{array}{l}
1.000 \frac{2,67 \times 2,30}{22,1}=278 \\
1.000 \frac{2,67 \times 2,30}{16,9}=363
\end{array}\right.
$$

siguiendo el ejemplo anterior. Estos valores dan lugar a diferencias entre la dosificación calculada $D_{\mathrm{M}}$ y las nominales (verdaderas) $\mathrm{D}$, de $-37 \mathrm{~kg} \mathrm{y}+48 \mathrm{~kg}$ en cada caso, lo que supone errores de $-12 \% \mathrm{y}+15 \%$, respectivamente, de acuerdo con lo encontrado anteriormente.

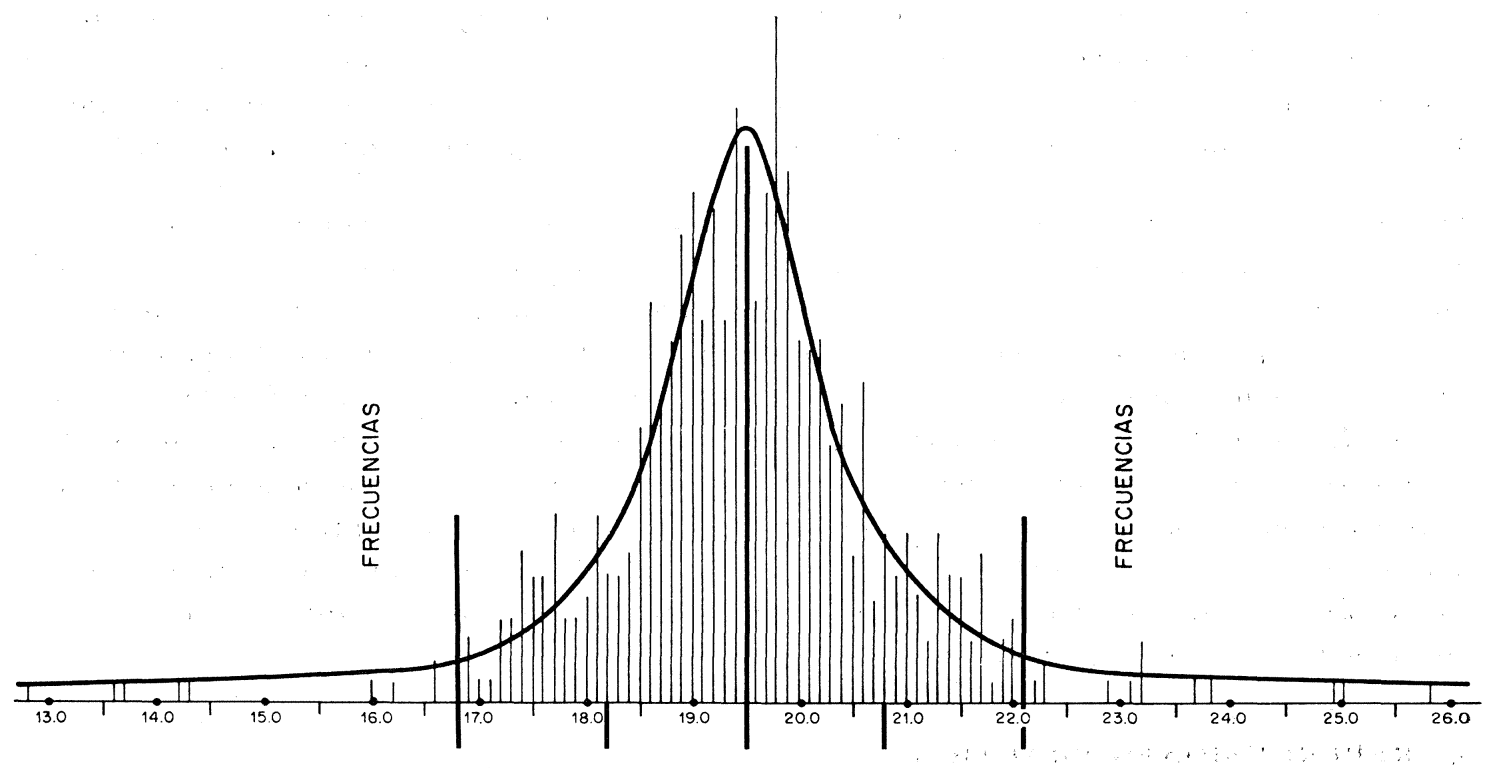

INTERVALOS DE CLASE, I Y VALORES CENTRALES, $x_{i}$

Fig. 1

Como mínimo, los citados errores pueden dar lugar a valores de dosificación comprendidos entre:

$$
\mathrm{D}_{\mathrm{M}}=1.000 \frac{2,67 \times 2,30}{19,5}=315
$$

y

$$
\mathrm{D}=\left\{\begin{array}{l}
1.000 \frac{2,67 \times 2,30}{20,8}=295 \\
1.000 \frac{2,67 \times 2,30}{18,1}=339
\end{array}\right.
$$

Estos valores dan lugar a diferencias entre la dosificación calculada $D_{M}$ y las nominales (verdaderas) $\mathrm{D}$, de $-20 \mathrm{~kg} \mathrm{y}+24 \mathrm{~kg}$ en cada caso, lo cual supone errores de $-6 \% \mathrm{y}$ $+8 \%$, respectivamente. En promedio, los errores serían del orden de $\pm 10 \%$. 
En el caso más desfavorable, el error es igual al resultante según la norma NELC 5.01-a; en los demás casos es menor.

Esto indica que la variabilidad de los cementos en cuanto a composición -y concretamente en cuanto a contenido de sílice- ha sido menor en el período 1963-1969, que lo fue en la época en que se estableció la norma NELC 5.01-a.

En cuanto a los valores aberrantes eliminados sucesivamente en la serie inicial de 601 , los que pertenecen a la cabeza de la serie, con contenidos de sílice soluble altos, pueden corresponder a cementos puzolánicos o siderúrgicos (o que contenían puzolana o escoria en alguna proporción), y que, por carecer de denominación especial, fueron considerados como portland. Los pertenecientes a la cola de la serie, con contenidos de sílice bajos, pueden corresponder a cementos diluidos, con adiciones de naturaleza caliza en proporción incluso superior al $10 \%$ que admite la norma española vigente (9), y que, por carecer también de denominaciones especiales, fueron considerados igualmente como portland. Puede tratarse, incluso, de cementos de adición C-150, no declarados como tales, o de cementos P-150, que en el período 1961-1964 estaban normalizados como portland (12).

En todo caso, es obvio que la estadística rechaza los valores correspondientes a estos cementos, lo cual es necesario para calcular el error relativo probable sobre la media de la serie retenida, que es el error que hay que considerar, aunque esta media no varíe prácticamente, en relación con la de la serie inicial completa, como indica la tabla 3. Este rechazo es además una evidencia indirecta de lo acertado que en su día fue la supresión de la categoría P-150 del tipo portland, y su sustitución por la categoría C-150 del tipo de adición, con las modificaciones consiguientes y, desde luego, sin la denominación ni la consideración de portland. Esta fue una de las principales mejoras introducidas en la norma vigente - versión de 1964 (9)—, en relación con la versión primitiva de 1961 (12). Pero es también una prueba indirecta de lo inadecuado que fue el mantener la extensión de la definición de cemento portland.

\section{REPERCUSIONES PRACTICAS}

Demostrado con un número suficientemente elevado de datos que el contenido de sílice soluble de los cementos españoles que con criterio amplio pueden llamarse portland, ha oscilado en los años de 1963 a 1969 entre 16,9\% y 22,1\%, y que su valor medio es indiscutiblemente de $19,5 \%$ (fig. 1), los resultados que se obtienen al utilizar como media el valor $20,5 \%$ de la norma NELC 5.01-a, o el valor 19,5 \%, más en consonancia con la realidad actual de los cementos españoles, se dan en las tablas 4 y 5 , respectivamente.

La comparación de ambas tablas en sus apartados $\mathrm{V}$ pone de relieve que el valor medio 19,5. \% está centrado y el 20,5 \% no lo está. En consecuencia, con el primero los errores positivos y negativos son simétricos y con el segundo no. Esto lleva consigo el que el empleo de una media de 20,5\% en lugar de 19,5\% perjudique a la dosificación de los hormigones hechos con cementos más pobres en sílice soluble, y beneficie a la de los hormigones con cementos más ricos en dicha sílice. Pero se da la circunstancia de que entre los cementos más pobres en sílice soluble se cuentan los que en alguna proporción contienen adiciones de tipo calizo, generalmente inertes, mientras que entre los más ricos en sílice soluble se encuentran los que en ciertas dosis contienen adiciones del tipo de las puzolanas o de las escorias, generalmente activas - siempre teniendo en cuenta la definición ampliada que de cemento portland da la norma española vigente (9)-. 
T A $\mathrm{B}$ L A 4

$\mathrm{D} \cdot \mathrm{S}_{\mathrm{c}}=2.300 \cdot \mathrm{S}_{\mathrm{h}}$

Dosificación nominal D del hormigón

$\mathbf{k g} / \mathbf{m}^{3}$

\begin{tabular}{|c|c|c|c|c|c|c|c|}
\hline \multirow[b]{2}{*}{ 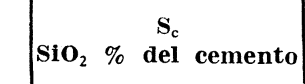 } & & & & & & & \\
\hline & 150 & 200 & 250 & 300 & 350 & 400 & 450 \\
\hline 16,9 & 2.535 & 3.380 & 4.225 & 5.070 & 5.915 & 6.760 & 7.605 \\
\hline 20,5 & 3.075 & 4.100 & 5.125 & 6.150 & 7.175 & 8.200 & 9.225 \\
\hline 22,1 & 3.315 & 4.420 & 5.525 & 6.630 & 7.735 & 8.840 & 9.945 \\
\hline
\end{tabular}

\begin{tabular}{|c|c|c|c|c|c|c|c|}
\hline \multirow[b]{2}{*}{$\begin{array}{lll} & \mathrm{S}_{\mathrm{c}} \\
\mathrm{SiO}_{2} & \% & \text { del } \\
\text { cemento }\end{array}$} & \multicolumn{7}{|c|}{ Sílice soluble $\mathbf{s}_{\mathrm{h}} \%$ del hormigón } \\
\hline & 150 & 200 & 250 & 300 & 350 & 400 & 450 \\
\hline 16,9 & 1,10 & 1,47 & 1,84 & 2,20 & 2,57 & 2,94 & 3,31 \\
\hline 20,5 & 1,34 & 1,78 & 2,23 & 2,67 & 3,12 & 3,57 & 4,01 \\
\hline 22,1 & 1,44 & 1,92 & 2,40 & 2,88 & 3,36 & 3,84 & 4,32 \\
\hline
\end{tabular}

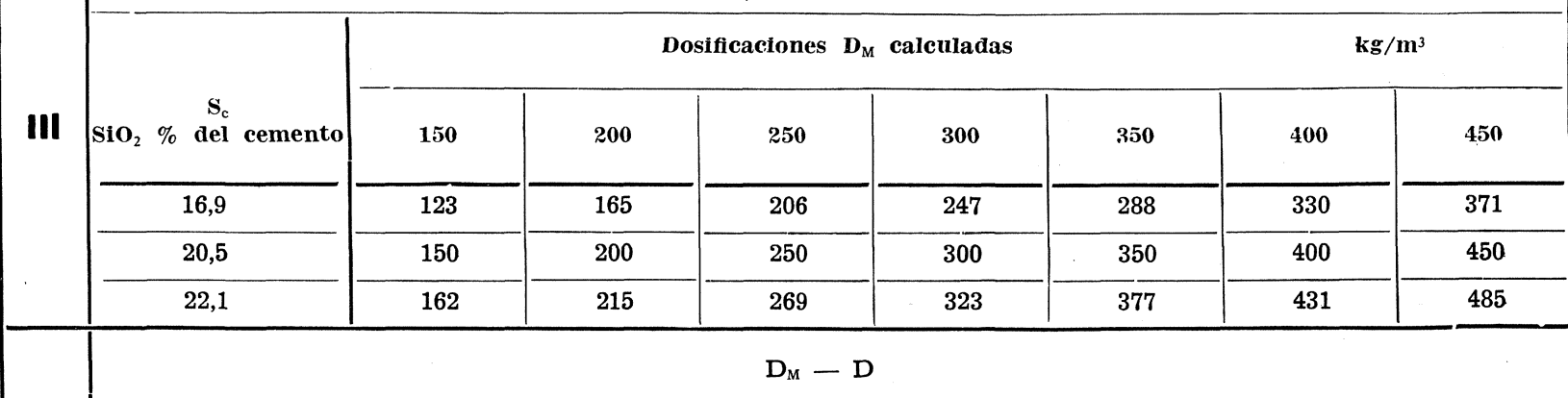

\begin{tabular}{|c|c|c|c|c|c|c|c|c|}
\hline \multirow{5}{*}{ IV } & & \multicolumn{7}{|c|}{ Diferencias $D_{M}-D$} \\
\hline & $\mathbf{S i O}_{2} \% \begin{array}{cc}\mathbf{S}_{\mathrm{c}} & \text { del } \\
\text { cemento }\end{array}$ & 150 & 200 & 250 & 300 & 350 & 400 & 450 \\
\hline & 16,9 & -27 & -35 & -44 & -53 & -62 & --70 & -79 \\
\hline & 20,5 & 0 & 0 & 0 & 0 & 0 & 0 & 0 \\
\hline & 22,1 & +12 & +15 & +19 & +23 & +27 & +31 & +35 \\
\hline \multirow{6}{*}{$\mathbf{v}$} & \multicolumn{8}{|c|}{$\mathrm{E} \%=100 \cdot \frac{\mathrm{D}_{\mathrm{M}}-\mathrm{D}}{\mathrm{D}}$} \\
\hline & & \multicolumn{7}{|c|}{ Errores por ciento E \% } \\
\hline & $\mathbf{S i O}_{2} \% \begin{array}{c}\mathrm{S}_{\mathrm{c}} \\
\text { del cemento }\end{array}$ & 150 & 200 & 250 & 300 & 350 & 400 & 450 \\
\hline & 16,9 & -18 & $-17,5$ & -18 & -18 & -18 & $-17,5$ & $-17,5$ \\
\hline & 20,5 & 0 & 0 & 0 & 0 & 0 & 0 & 0 \\
\hline & 22,1 & +8 & $+7,5$ & +8 & +8 & +8 & +8 & +8 \\
\hline
\end{tabular}


T A B L A 5

$\mathrm{D} \cdot \mathrm{S}_{\mathrm{c}}=2.300 \cdot \mathrm{S}_{\mathrm{h}}$

Dosificación nominal D del hormisón

$\mathbf{k g} / \mathbf{m}^{3}$

I $\quad \mathrm{SiO}_{2} \% \begin{array}{cc}\mathbf{S}_{\mathrm{c}} \\ \text { del cemento }\end{array}$

\begin{tabular}{c|c|c|c|c|c|c}
\hline 150 & 200 & 250 & 300 & 350 & 400 & 450 \\
\hline 2.535 & & & & & \\
\hline 2.925 & 3.380 & 4.225 & 5.070 & 5.915 & 7.760 & 7.605 \\
\hline 3.315 & 3.900 & 4.875 & 5.850 & 6.825 & 7.800 & 8.775 \\
\hline 4.420 & 5.525 & 6.630 & 7.735 & 8.840 & 9.945 \\
\hline
\end{tabular}

$\mathbf{S}_{\mathbf{h}}=\mathrm{D} \cdot \frac{\mathbf{S}_{\mathrm{c}}}{2.300}$

Sílice soluble $S_{h} \%$ del hormigón

\begin{tabular}{|c|c|c|c|c|c|c|c|}
\hline $\mathrm{SiO}_{2} \%$ del cemento & 150 & 200 & 250 & 300 & 350 & 400 & 450 \\
\hline 16,9 & 1,10 & 1,47 & 1,84 & 2,20 & 2,57 & 2,94 & 3,31 \\
\hline 19,5 & 1,27 & 1,70 & 2,12 & 2,54 & 2,97 & 3,39 & 3,82 \\
\hline 22,1 & 1,44 & 1,92 & 2,40 & 2,88 & 3,36 & 3,84 & 4,32 \\
\hline
\end{tabular}

Dosificaciones $\mathbf{D}_{\mathrm{M}}$ calculadas

$\mathrm{kg} / \mathrm{m}^{3}$

\begin{tabular}{|c|c|c|c|c|c|c|c|}
\hline $\mathrm{SiO}_{2} \% \frac{\mathrm{S}_{\mathrm{c}}}{\mathrm{del} \text { cemento }}$ & 150 & 200 & 250 & 300 & 350 & 400 & 450 \\
\hline 16,9 & 130 & 173 & $217_{1}$ & 259 & 303 & 347 & 390 \\
\hline 19,5 & 150 & 200 & 250 & 300 & 350 & 400 & 450 \\
\hline 22,1 & 170 & 226 & 283 & 340 & 396 & 453 & 509 \\
\hline
\end{tabular}

Diferencias $D_{M}-\mathbf{D}$

\begin{tabular}{|c|c|c|c|c|c|c|c|c|}
\hline \multirow[t]{4}{*}{ IV } & 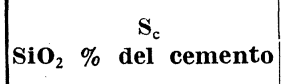 & 150 & 200 & 250 & 300 & 350 & 400 & 450 \\
\hline & 16,9 & -20 & -27 & -33 & -41 & -47 & -53 & -60 \\
\hline & $\overline{19,5}$ & 0 & 0 & 0 & 0 & 0 & 0 & 0 \\
\hline & $\overline{22,1}$ & +20 & +26 & +33 & +40 & +46 & +53 & +59 \\
\hline
\end{tabular}

Errores por ciento $\mathbf{E} \%$

\begin{tabular}{|c|c|c|c|c|c|c|c|}
\hline $\begin{array}{lll} & \mathbf{S}_{\mathrm{c}} \\
\mathbf{S i O}_{2} & \% & \text { del cemento }\end{array}$ & 150 & 200 & 250 & 300 & 350 & 400 & 450 \\
\hline 16,9 & -13 & $-13,5$ & -13 & -14 & -13 & -13 & -13 \\
\hline 19,5 & 0 & 0 & 0 & 0 & 0 & 0 & 0 \\
\hline 22,1 & +13 & +13 & +13 & +13 & +13 & +13 & +13 \\
\hline
\end{tabular}


El empleo del valor 19,5 \% como media más real en la actualidad trata por igual (o al menos más equitativamente) a unos y otros cementos, implicando al mismo tiempo la comisión de un error menor que el máximo cometido en el caso de aceptar la media de $20,5 \%$.
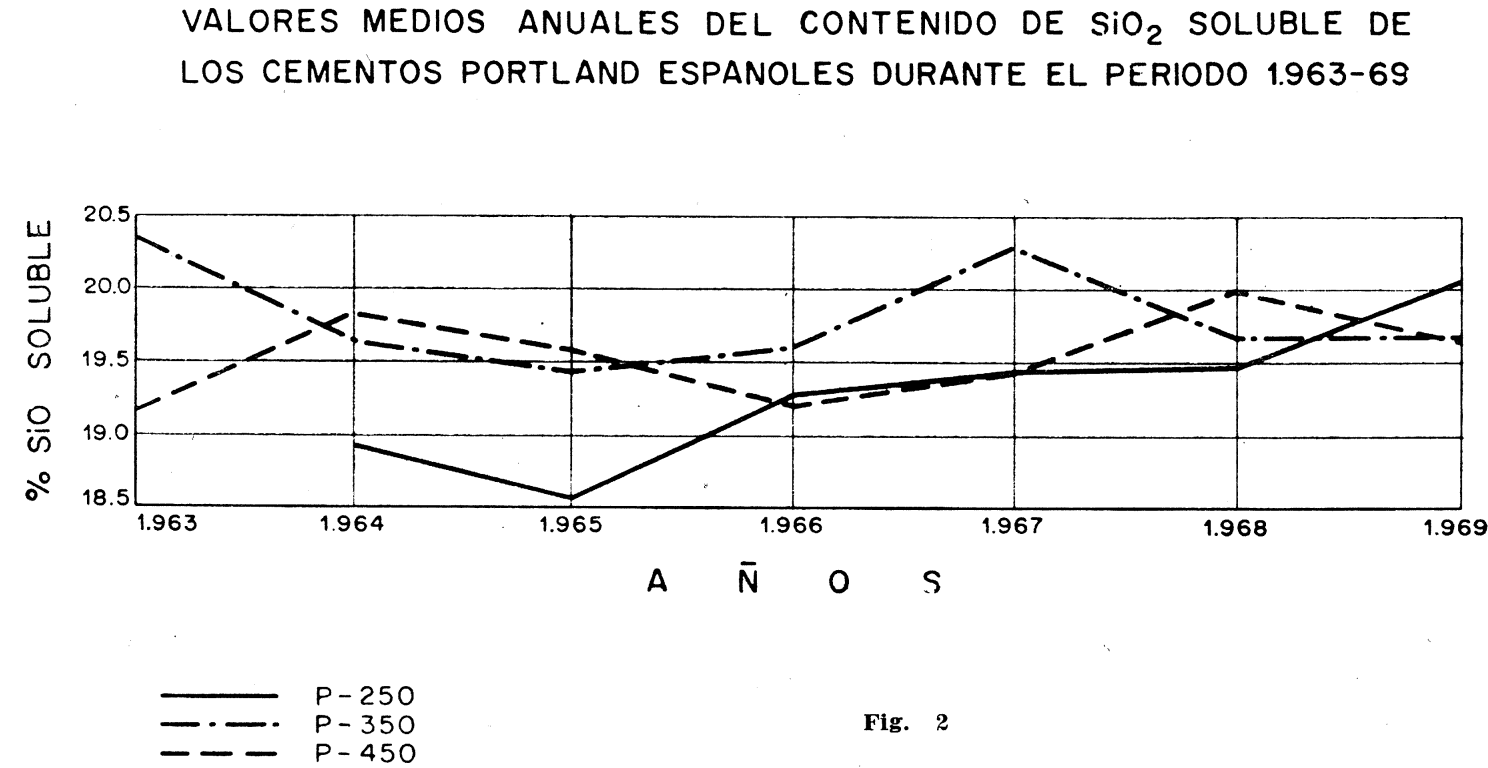

Fig. 2

La diferencia de errores es del orden del $5 \%$, como se puede apreciar comparando las tablas $4-\mathrm{V}$ y $5-\mathrm{V}$.

En definitiva, el error máximo que se puede cometer al utilizar 19,5\% como valor medio, es del orden de $\pm 13 \%$, es decir, análogo al que por idéntico concepto admite tácitamente la norma NELC 5.01-a, y menor del que se comete manteniendo el valor medio de $20,5 \%$. Este error es, además simétrico, por ser la nueva media, la media real del intervalo de valores que en la práctica se presentan como individuos en una distribucion normal de Gauss-Laplace (media centrada).

Por lo tanto, se propone y recomienda la adopción del valor 19,5 \% como media del contenido de sílice soluble de los cementos portland españoles actuales, a todos los efectos de la norma NELC 5.01-a.

\section{CONCLUSIONES}

1. La norma NELC 5.01-a (2) para la dosificación de cemento en hormigones y morteros fraguados, hechos con cemento portland, era aplicable siempre, en las condiciones que estipula, cuando regía la antigua norma española para cementos (8).

2. La norma NELC 5.01-a (2) en rigor no es siempre aplicable para tal fin, en vista de la ampliación de la definición de cemento portland introducida en las sucesivas nuevas normas españolas para cementos (9) (12).

3. En consecuencia, el cemento portland queda, en general, sujeto a las mismas limitaciones que la norma NELC 5.01-a (2) fija para otros cementos, puzolánicos o siderúrgicos. 
4. Las adiciones no silícicas no impiden la determinación de la dosificación verdadera de material hidráulicamente activo en un mortero u hormigón. La dosificación hallada es menor que la correspondiente de material añadido en concepto de "conglomerante" (cemento más adición). La diferencia entre ambas dosificaciones puede ser del orden del $10 \%$, si el "conglomerante" se ajusta a la definición ampliada de cemento portland dada en la norma española vigente para cemento (9); mayor, si no se ajusta a ella.

5. Las adiciones silícicas impiden la dosificación verdadera de cemento portland, pudiendo dar lugar a errores, poco probables, eso sí, del orden del $35 \%$ al $40 \%$.

6. Una estadística de los cementos portland o innominados hecha en el Departamento de Química del I.E.T.c.c., extensiva a 600 cementos, y que abarca los años 1963 a 1969, ambos inclusive, pone de relieve que los contenidos de sílice soluble responden a una distribución normal de Gauss-Laplace, con valores extremos de $17 \%$ y $22 \%$, $\mathrm{y}$ valor medio de $19,5 \% \pm 0,1$.

7. El error máximo que se puede cometer al utilizar este valor medio, a efectos de la norma NELC 5.01 -a, es del orden del $13 \%$, que sumado al $4 \%$ propio del método, puede dar, en conjunto, un error de $\pm 17 \%$, del mismo orden que el señalado en dicha norma, y menor que si se utiliza el $20,5 \%$ aún vigente.

8. El valor medio $19,5 \%$ es centrado respecto de la amplia serie real de valores, como corresponde a una media verdadera, lo que no sucede con el valor 20,5\%. Esto hace que al utilizar este último valor en lugar del verdadero, resulten discriminados los hormigones a los que se aplica la norma NELC 5.01-a, en el sentido de verse perjudicados (por dosificaciones más bajas) los hormigones hechos con cementos pobres en sílice soluble (con adiciones inertes no silíceas - véase conclusión 4-) y favorecidos (por dosificaciones más altas que las verdaderas) los hormigones fabricados con cementos ricos en sílice soluble (con adiciones de escorias o puzolanas -véase conclusión 5-).

9. En vista de cuanto antecede, y como conclusión indirecta, se confirma el acierto que supuso, en su momento, y de cara a la realidad, suprimir en la norma española (12) la ínfima categoría de cemento portland P-150, y sustituirla en la vigente norma actual (9) por el nuevo tipo de cemento C-150 de adición (no portland). En esta misma línea es de lamentar la ampliación que de la definición de cemento portland (sin más especificación) se introduja en la moderna norma española (12) para cemento, la cual se mantiene en la actualmente vigente.

10. Se propone utilizar en lo sucesivo, y mientras otros argumentos análogos a los expuestos en este trabajo no aconsejen otra cosa, el valor de $19,5 \%$ como contenido medio de sílice soluble de los cementos portland españoles en la actualidad, a todos los efectos de la norma NELC 5.01-a.

\section{referencias}

(1) Calleja, J. "Observaciones acerca de la dosificación de cementos en morteros y hormigones fraguados”. Materiales de Construcción-Ultimos Avances I.E.T.c.c., núm. 121, enero-febrero-marzo 1966.

(2) NELC 5.01-a (Normas de Ensayo del Laboratorio Central). "Determinación de la dosificación de hormigones y morteros fraguados con cemento". Publicación núm. 42 del Laboratorio Central para el Ensayo de Materiales de Construcción. Madrid, 1947.

(3) Instrucción para el Proyecto y Ejecución de Obras de Hormigón (B. O. del Estado de 1 de julio de 1944). 
(4) Instrucción para el Proyecto y Ejecución de Obras de Hormigón en Masa o Armado. Ministerio de Obras Públicas: Decreto de la Presidencia de Gobierno, núm. 2.987, de 20 de septiembre de 1968

(5) ASTM, C-85-66: Standard Method for "Cement Content of Hardened Portland Cement Concrete".

(6) CEMBUREAU: Sub-Committee for "Analysis of Hardened Concrete"; Spanish member: Dr. J. CALLEJA (I.E.T.c.c.).

(7) RUIZ DE GAUnA, A. Tesis doctoral: "Contribución al estudio de los Morteros y Hormigones Fraguados: Nuevo procedimiento de dosificación con aplicación de computadores digitales". En trámite y vías de publicación. Director de Tesis: Dr. Jose CAlleJa. Madrid, 1970

(8) Pliego de Condiciones para la Recepción de los Aglomerantes Hidráulicos en las Obras de Carácter Oficial. B. O. de 25 de febrero, 1930.

(9) Pliego de Condiciones para la Recepción de Conglomerantes Hidráulicos en las Obras de Carácter Oficial. B. O. del Estado núm. 109 de 6 de mayo de 1964 y 222 de 15 de septiembre de 1964: PCCH64, “Manuales y Normas" del I.E.T.c.c., Madrid 1964.

(10) ASTM, C-114-67: Standard Methods for "Chemical Analysis of M Hydraulic Cement".

(11) ASTM, C-85-42 : Standard Method for "Cement Content of Hardened Portland Cement Concrete".

(12) PCCH-61, “Manuales y Normas" del I.E.T.c.c., Madrid 1961. 